Journal of Innovative Image Processing (JIIP) (2020)

Vol.02/ No. 01

Pages: $35-43$

https://www.irojournals.com/iroiip/

DOI: https://doi.org/10.36548/jiip.2020.1.004

\title{
Mellowness Detection of Dragon Fruit Using Deep Learning Strategy
}

\author{
Dr. T. Vijayakumar, \\ Professor, \\ Department of ECE, \\ GNIT, Hyderabad, India. \\ Email id: vishal16278@yahoo.co.in
}

\author{
Mr. R. Vinothkanna, \\ Department of ECE, Vivekanandha College of Technology for Women, \\ Namakal, India. \\ Email: rvinothkannaphd@gmail.com
}

\begin{abstract}
The agriculture being a main source of income in many developing countries such as India, Indonesia, etc. The economic development of these countries depends on the GDP (Gross Domestic Progress) rate of the agricultural products. However due to miscalculations in the maturity of the fruits and vegetables leads to the wastage of foods. In general many measure were taken to minimize the food spoilage and by tracking the each stage of the vegetables and fruits carefully, but resulted in a hefty human labor, and weariness. Specifically the non-climacteric fruit such as the dragon fruit requires much attention as it is has to be harvested after it is ripened and cannot be ripened after harvesting using the hastening ripening process such as the ethylene, carbide, and $\mathrm{CO}_{2}$ etc. So the paper has put forth the application to identify the mellowness in the dragon fruit using the RESNET 152 a deep learning convolution neural network to identify the dragon fruits mellowness and it's time to harvest. The model was trained using the python and the tensor flow. The developed structure was trained using the pictures of the dragon fruit in the different stages of its mellowness and was tested using the region of convergence and the confusion matrix with 100 new data. The testing was carried with the different number of epoch ranging from 10 to 500. The results obtained were more accurate compared to the VGG16/19 in the terms of Accuracy and loss in training and testing.
\end{abstract}

Keywords: Mellowness Detection, Harvesting, Dragon Fruit, Deep Convolution Neural Network, RESNET152

\section{Introduction}

The dragon fruit are very prominent since the year 2000, it is a non-climacteric fruit that has to be harvested after it has attained the mellowness as it could not be ripened by the hastening ripening process such as the ethylene, carbide, and $\mathrm{CO}_{2}$ etc. a keen observation is required in its each stage to correctly note down the mellowness of the fruit and its time of harvesting. The dragon fruit are especially grown in regions like 
Journal of Innovative Image Processing (JIIP) (2020)

Vol.02/ No. 01

Pages: $35-43$

https://www.irojournals.com/iroiip/

DOI: https://doi.org/10.36548/jiip.2020.1.004

Jember, Bali, Malang and Pasuruan in the Indonesian country and are exported to wide part of the world. So the vegetation of Dragon fruit has an important part in increasing the GDP of the Indonesia.

So much attention are in the each stage of the fruits growth as the delayed harvesting or the early harvesting of the fruit would lead to spoilage of the food and also affect their economy growth of the country. The ripened fruit can be harvested and protected to retain the fruit quality. The farmers are still utilizing the manual methods and efforts to use to follow the fruits in it each stage of maturity and identify its ripeness an sometimes even miscalculate the ripeness level and harvest the fruits before ripeness, so the heft manual work put forth by the farmer results in the weariness and boredom. They have to keenly note down the each step of development of the dragon fruit and starts counting the actual time to harvest from the day the flowers are developed in the trees of the dragon fruit.

So the proposed method in paper devises an application using the RESNET 152 a deep learning convolution neural network to learn every stage of the fruits development and classify the ripened fruit from the unripen and as well sort out the time to harvest the fruit such that its quality could be retained without any damage.

The RESNET 152 model is built with the capability of classifying the dataset based on the labels provided during training process that enables the model to learn the extracted features from the images subjected to training, the training process is repeated with different set of images to have a perfect classification with higher accuracy. The data subjected for testing is examined in accordance with certain attributes of the every label based on the result acquired from the training process.

The paper utilizing the deep CNN for classifying is planned with the main headings such as related works in 2 , the proposed work in 3 , the results evaluation in 4 and conclusion in 5

\section{Related works}

To have a clear insight regarding the mellowness of the dragon fruit many methods have been developed, and many researches are still under development some of the developed methods to have a deep knowledge on the ripeness of the dragon fruit, was gained from the below mentioned works of different researchers, the ripened degree of the fruit was and readiness to harvesting and the methods to store Hylocereus fruit, was developed by the author Le Bellec, et al [1] in the year 2006.

The author Istianingsih, et al [2] developed the method to identify the proper timing of the harvesting and the perfect storage temperature and highlighted the correlation that existed between the time of harvest, 
storage, freshness, cumulative yield loss and hardness etc. Further the mellowness deduction was performed with the tool developed based on the HSV segmentation method put forth by the author Wismadi, I et al [3] , the results observed through this method proved that it was time consuming and less accurate as it was able to achieve only $<85 \%$ accuracy, more over the utilization of the color features to classify the fruits maturity is an disadvantage as the fruits could not be checked in their natural stage.

Many studies and survey by Khrisne, D. C., et al [4] and Simonyan, et al [5] where developed for have useful insights on detecting the time to harvest the fruits. From the survey it was found that the deep learning methods were highly useful in finding out the mellowness of the dragon fruit, further the author Raj, Jennifer S. et al [6] performed the "A Comprehensive Survey on the Computational Intelligence Techniques and Its Applications." Joseph, S. Iwin Thanakumar et al [7] performed the "Survey of data mining algorithms for intelligent computing system."

Koresh, M. H., and J. Deva et al [8] conducted the "Computer vision based traffic sign sensing for smart transport." Manoharan, Samuel et al [9] did the "A smart image processing algorithm for text recognition, information extraction and vocalization for the visually challenged." Chandy, Abraham et al [10] performed the "RGBD Analysis for Finding the Different Stages of Maturity of Fruits in Farming." J. Vijitha Ananthi et al [11] proposed the " Automation Using Iot in Greenhouse Environment." Raj, Jennifer S., and J. Vijitha Ananthi et al [12] performed the "Recurrent Neural Networks and Nonlinear Prediction in Support Vector Machines." Samuel et al [13] conducted the "Study on Hermitian Graph Wavelets in Feature Detection."

\section{Proposed Work}

The work utilizes the deep convolutional neural network to categorize and identify the dragon fruit that reached it mellowness stages and that is ready for gleaning. The method utilizes the Residual Network that is shortly termed as the RESNET to perform the task. This was introduced by Microsoft as the remedy for the problems aroused in the deep networks due to the process of training. Some of the issues faced were (i) the degradation caused in the convergence of the deeper networks, resulting in accuracy saturation followed by degradation and (ii) error in training. (iii) The optimization of the deeper networks were not much easy.

The residual layer eluded the hoping of each small piled layers in order to straightforwardly fitting the sought after fundamental mapping. The layers are explicitly let to fit into the residual mapping. The $F(X)+$ $X$ shown in the figure. 1 below is achieved using the feed forward neural networks that has short links. These short links help in jumping from one layer to the next and also in sorting out the mapping, further the outputs obtained are added to the piled layers output. 
Journal of Innovative Image Processing (JIIP) (2020)

Vol.02/ No. 01

Pages: $35-43$

https://www.irojournals.com/iroiip/

DOI: https://doi.org/10.36548/jiip.2020.1.004

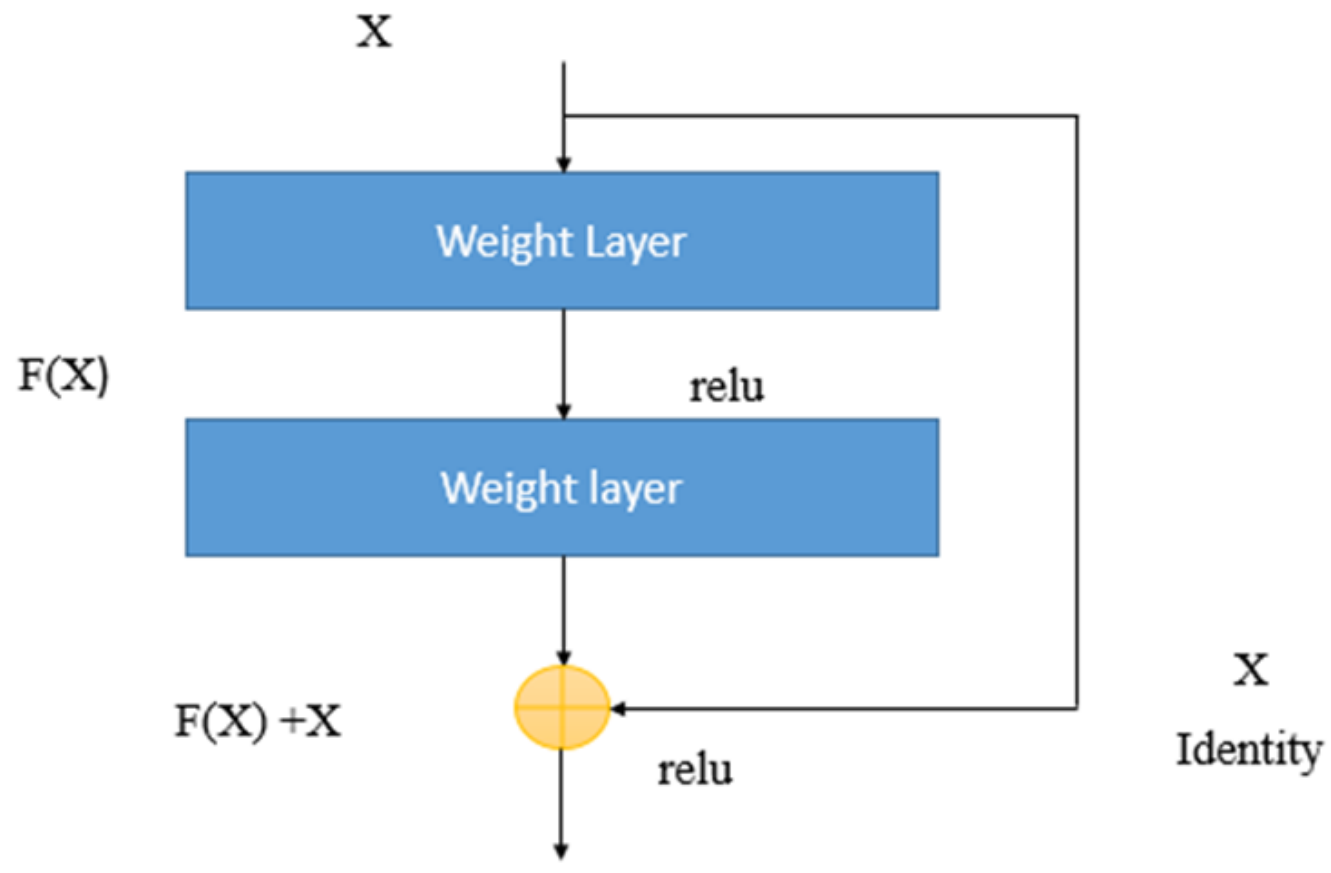

Figure.1 Residual Network

The benefits of the RESNET work is it is easy optimize and has higher accuracy when the depth is increased unlike the previous network that results in degradation and saturated accuracy. The residual networks are similar to plain networks but has shorts links inserted to it, this makes the network into the counterpart residual network. The RESNET networks is encompassed with much fewer filters and low complexity compared to the VGGNETs

The residual network uses the short links directly if the inputs and the outputs are of similar dimensions and does the identify mapping and more zero entries to increase the dimensions as well as utilizes a projection shortcut to match the dimension if the input and the output has different dimensions. For the feature map with size two the operations the short links are performed in two strides. 
Journal of Innovative Image Processing (JIIP) (2020)

Vol.02/ No. 01

Pages: $35-43$

https://www.irojournals.com/iroiip/

DOI: https://doi.org/10.36548/jiip.2020.1.004

\begin{tabular}{|l|l|l|}
\hline Layer Name & Output Size & \multicolumn{1}{|c|}{152 Layer } \\
\hline Conv1 & $112 \times 112$ & $7 \times 7,64$, stride 2 \\
\hline Conv2.x & $56 \times 56$ & $\begin{array}{l}3 \times 3 \text { max pool stride 2 } \\
{\left[\begin{array}{c}1 \times 1,64 \\
3 \times 3,64 \\
1 \times 1,256\end{array}\right] \times 3}\end{array}$ \\
\hline Conv3.x & $28 \times 28$ & {$\left[\begin{array}{l}1 \times 1,128 \\
3 \times 3,128 \\
1 \times 1,512\end{array}\right] \times 8$} \\
\hline Conv4.x & $14 \times 14$ & {$\left[\begin{array}{c}1 \times 1,256 \\
3 \times 3,256 \\
1 \times 1,1024\end{array}\right] \times 36$} \\
\hline Conv5.x & $7 \times 7$ & {$\left[\begin{array}{c}1 \times 1,512 \\
3 \times 3,512 \\
1 \times 1,2048\end{array}\right] \times 3$} \\
\hline Flops & & Average pool, 1000-dc,softmax \\
\hline & & $11.3 \times 10^{8}$ \\
\hline
\end{tabular}

Table.1. RESNET 152 Layer

The proposed method uses the RESNET 152-layer using more three blocks layer as shown in above table.1. Though the depth of the RESNET is increased it still has a lower complexity than the VGG16/ 19

The training process is done with the images that are manually categorized as fruit with mellowness or ready for harvesting and not ready for harvesting, this was done with the help of experts. Every image used in training was labelled according to the experts categorization, further the feature extracted was used in the in training the RESNET 152. The pictures in the figure. 2 below shows the picture of mellowness fruit that is ready for harvest. 
Journal of Innovative Image Processing (JIIP) (2020)

Vol.02/ No. 01

Pages: $35-43$

https://www.irojournals.com/iroiip/

DOI: https://doi.org/10.36548/jiip.2020.1.004

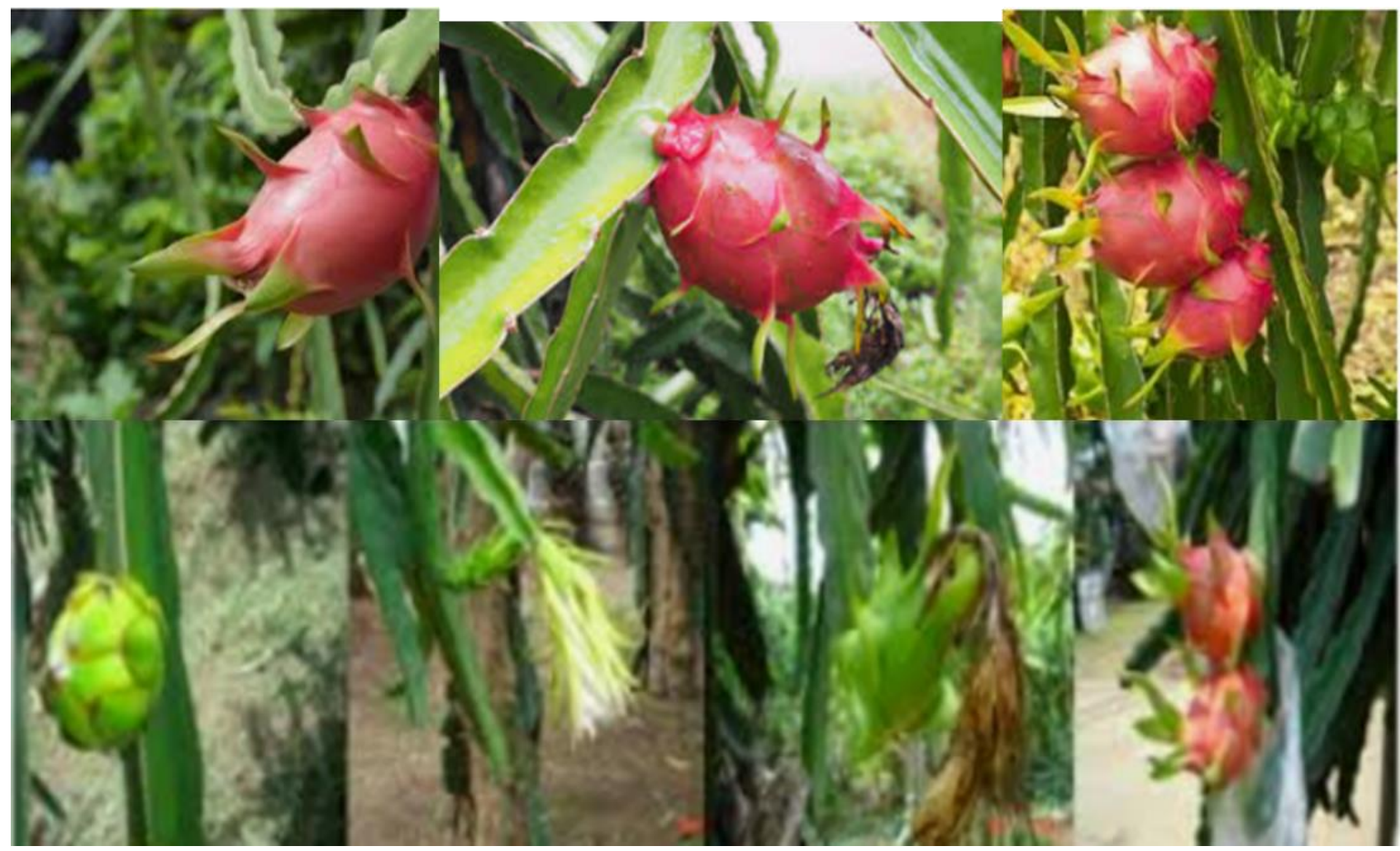

Figure.2 Samples of Data set used in Training

Once the testing process is completed the model is tested to predict whether the fruit are ripe or unripe.

\section{Results Evaluation}

Eighty percent of the image set was used in training and twenty percent was used in testing after several iterations of training and testing the validation results for the proposed model was obtained. The process was performed with various Epochs ranging from 10 to 500 the results displayed in figure. 3 below are the training loss and accuracy for 10, 20, 50, 100 and 500 epochs. 
Journal of Innovative Image Processing (JIIP) (2020)

Vol.02/ No. 01

Pages: $35-43$

https://www.irojournals.com/iroiip/

DOI: https://doi.org/10.36548/jiip.2020.1.004

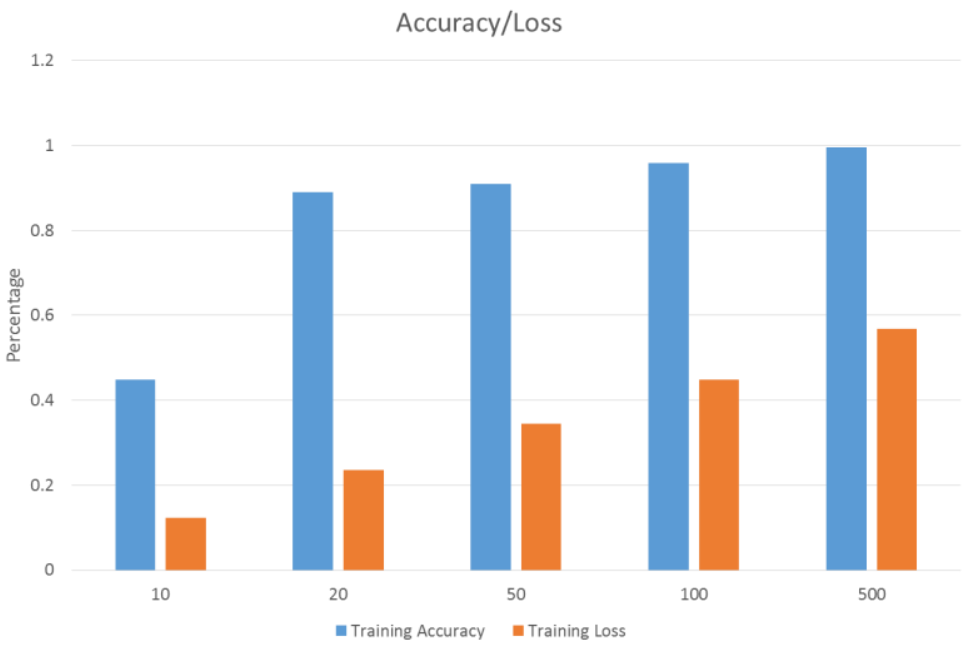

Figure.3 Training Accuracy and Loss

Based on the results acquired in the process of training, it was noted down that the proposed method had a higher learning accuracies, even with the number of epochs increases, unlike VGGNET that experiences a lesser accuracy when number of epochs increases.

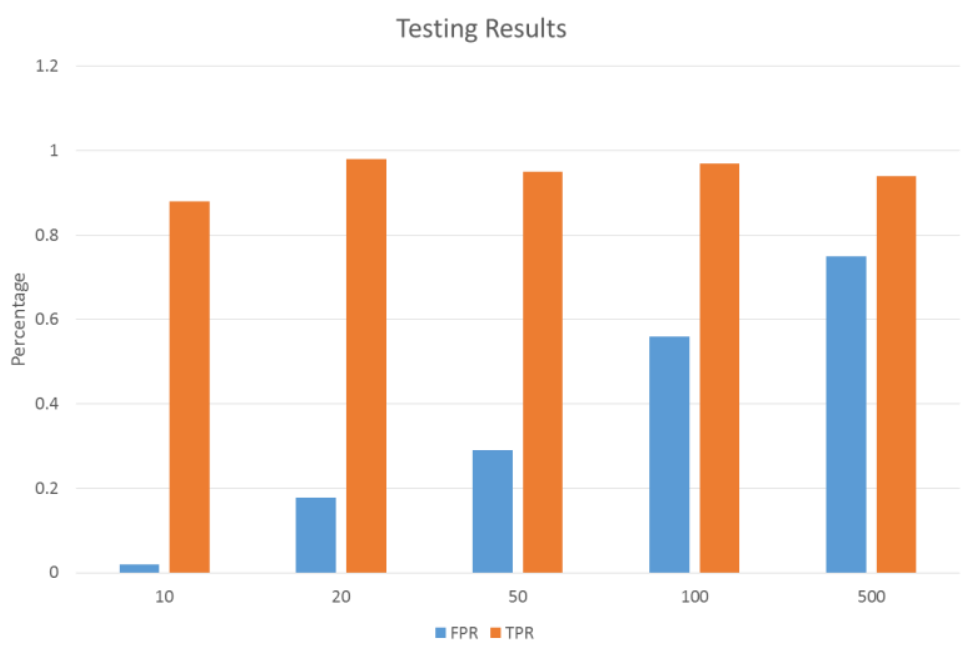

Figure.4 Testing Results 
Journal of Innovative Image Processing (JIIP) (2020)

Vol.02/ No. 01

Pages: $35-43$

https://www.irojournals.com/iroiip/

DOI: https://doi.org/10.36548/jiip.2020.1.004

The figure.4above shows the testing results obtained for the epoch's numbers that ranges from 10 to 500 . From the confusion matrix test results for the epochs, the TPR and the FPR value where attained and the ROC was formed as shown in figure. 5

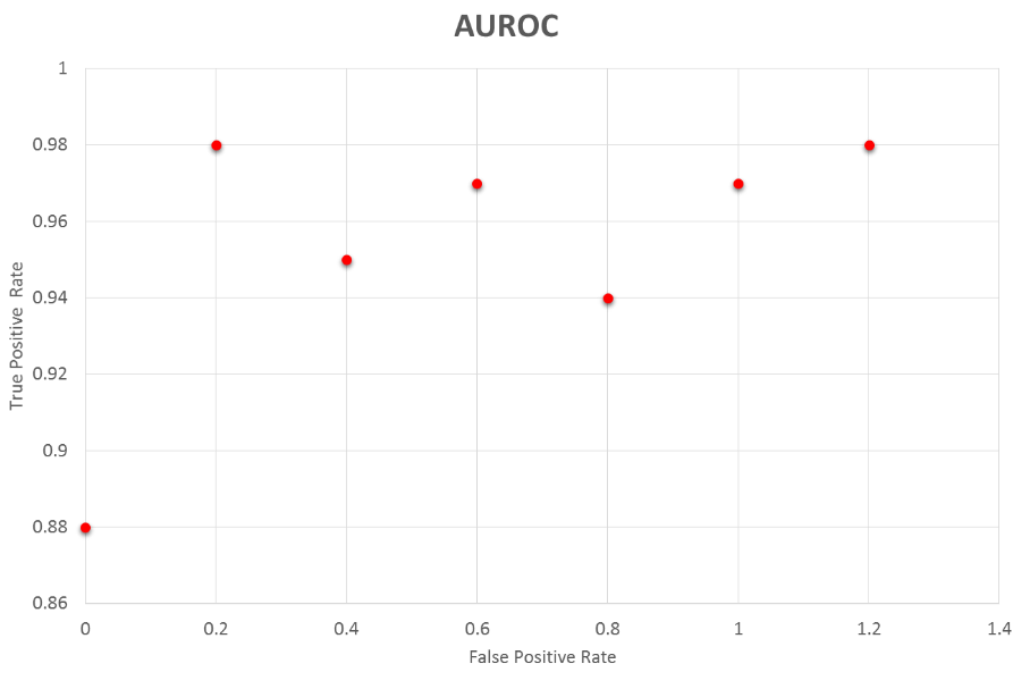

Figure.5 ROC Curve

The Trapezoidal method was used in estimating the AUROC and the it was concluded that the AUROC level of truth on the performance of the proffered method was measured to 1.0 stating the method to be very good compared to VGG $16 / 19$.

\section{Conclusion}

The RESNET 152 deep CNN based model was developed to identify the mellowness of the dragon fruit and the training and the testing process was done using the live image gathered on the different stages of the dragon fruit. The results observed proved to have a better accuracy in training and testing even in increased number of epochs, unlike the VGGNET were the accuracy gets degraded as the network become deeper and the number of epochs increases. The AUROC based on the test results was observed to be 1.0 which proves that proposed model does a better prediction than the prevailing. 
Journal of Innovative Image Processing (JIIP) (2020)

Vol.02/ No. 01

Pages: $35-43$

https://www.irojournals.com/iroiip/

DOI: https://doi.org/10.36548/jiip.2020.1.004

\section{References}

[1] Le Bellec, Fabrice, Fabrice Vaillant, and Eric Imbert. "Pitahaya (Hylocereus spp.): a new fruit crop, a market with a future." Fruits 61, no. 4 (2006): 237-250.

[2] Istianingsih, Tri, and Darda Efendi. "Pengaruh umur panen dan suhu simpan terhadap umur simpan buah naga super red (Hylocereus costaricensis)." Jurnal Hortikultura Indonesia 4, no. 1 (2015): 54-61.

[3] Wismadi, I. Made, Duman Care Khrisne, and I. Made Arsa Suyadnya. "Detecting the Ripeness of Harvest-Ready Dragon Fruit using Smaller VGGNet-Like Network." Journal of Electrical, Electronics and Informatics 3, no. 2: 35-38.

[4] Khrisne, D. C., and I. MA Suyadnya. "Indonesian Herbs and Spices Recognition using Smaller VGGNet-like Network." In 2018 International Conference on Smart Green Technology in Electrical and Information Systems (ICSGTEIS), pp. 221-224. IEEE, 2018.

[5] Simonyan, Karen, and Andrew Zisserman. "Very deep convolutional networks for large-scale image recognition." arXiv preprint arXiv:1409.1556 (2014).

[6] Raj, Jennifer S. "A Comprehensive Survey on the Computational Intelligence Techniques and Its Applications." Journal of ISMAC 1, no. 03 (2019): 147-159.

[7] Joseph, S. Iwin Thanakumar, and Iwin Thanakumar. "Survey of data mining algorithm's for intelligent computing system." Journal of trends in Computer Science and Smart technology (TCSST) 1, no. 01 (2019): 14-24.

[8] Koresh, M. H., and J. Deva. "Computer vision based traffic sign sensing for smart transport." J. Innovative Image Process.(JIIP) 1, no. 01 (2019): 11-19.

[9] Manoharan, Samuel. "A smart image processing algorithm for text recognition, information extraction and vocalization for the visually challenged." Journal of Innovative Image Processing (JIIP) 1, no. 01 (2019): 31-38.

[10] Chandy, Abraham. "RGBD Analysis for Finding the Different Stages of Maturity of Fruits in Farming." Journal of Innovative Image Processing (JIIP) 1, no. 02 (2019): 111-121.

[11] Raj, Jennifer S., and J. Vijitha Ananthi. "Automation Using Iot in Greenhouse Environment." Journal of Information Technology 1, no. 01 (2019): 38-47.

[12] Raj, Jennifer S., and J. Vijitha Ananthi. "Recurrent Neural Networks and Nonlinear Prediction in Support Vector Machines." Journal of Soft Computing Paradigm (JSCP) 1, no. 01 (2019): 33-40.

[13] Manoharan, Samuel. "Study On Hermitian Graph Wavelets in Feature Detection." Journal of Soft Computing Paradigm (JSCP) 1, no. 01 (2019): 24-32. 